\title{
Correction to: The global summit on the efficacy and effectiveness of spinal manipulative therapy for the prevention and treatment of non-musculoskeletal disorders: a systematic review of the literature
}

Pierre Côté ${ }^{1,2,3,4^{*}}$, Jan Hartvigsen ${ }^{5,6}$, Iben Axén ${ }^{7,8}$, Charlotte Leboeuf-Yde ${ }^{9}$, Melissa Corso ${ }^{1,2}$, Heather Shearer ${ }^{2,4}$, Jessica Wong ${ }^{1,2,3}$, Andrée-Anne Marchand ${ }^{10}$, J. David Cassidy ${ }^{3}$, Simon French ${ }^{11}$, Gregory N. Kawchuk ${ }^{12}$, Silvano Mior ${ }^{2,13}$, Erik Poulsen ${ }^{5}$, John Srbely ${ }^{14}$, Carlo Ammendolia ${ }^{4,15}$, Marc-André Blanchette ${ }^{10}$, Jason W. Busse ${ }^{16}$, André Bussières ${ }^{10,17}$, Carolina Cancelliere ${ }^{1,2}$, Henrik Wulff Christensen ${ }^{6}$, Diana De Carvalho ${ }^{18}$, Katie De Luca ${ }^{11}$, Alister Du Rose ${ }^{19}$, Andreas Eklund ${ }^{7}$, Roger Engel ${ }^{11}$, Guillaume Goncalves ${ }^{20}$, Jeffrey Hebert ${ }^{21}$, Cesar A. Hincapié ${ }^{22}$, Maria Hondras ${ }^{23}$, Amanda Kimpton ${ }^{24}$, Henrik Hein Lauridsen ${ }^{5}$, Stanley Innes ${ }^{25}$, Anne-Laure Meyer ${ }^{20}$, David Newell ${ }^{26}$, Søren O'Neill ${ }^{9,27}$, Isabelle Pagé ${ }^{10}$, Steven Passmore ${ }^{28}$, Stephen M. Perle ${ }^{29}$, Jeffrey Quon ${ }^{30}$, Mana Rezai ${ }^{2}$, Maja Stupar ${ }^{13}$, Michael Swain ${ }^{11}$, Andrew Vitiello ${ }^{31}$, Kenneth Weber ${ }^{32}$, Kenneth J. Young ${ }^{33}$ and Hainan $\mathrm{Yu}^{1,2}$

Correction to: Chiropr Man Therap 19, 348 (2021) https://doi.org/10.1186/s12998-021-00362-9

Following publication of the original article [1], we were notified of a typo in the last name of the 43rd author.

- Originally published name: Andrew Vitello

- Corrected name: Andrew Vitiello

The original article has been corrected.

The original article can be found online at https://doi.org/10.1186/s12998 021-00362-9.

* Correspondence: pierre.cote@uoit.ca

${ }^{1}$ Faculty of Health Sciences, Ontario Tech University, Oshawa, Canada

${ }^{2}$ Centre for Disability Prevention and Rehabilitation at Ontario Tech University and CMCC, Oshawa, Canada

Full list of author information is available at the end of the article

\section{Author details}

${ }^{1}$ Faculty of Health Sciences, Ontario Tech University, Oshawa, Canada. ${ }^{2}$ Centre for Disability Prevention and Rehabilitation at Ontario Tech University and CMCC, Oshawa, Canada. ${ }^{3}$ Division of Epidemiology, Dalla Lana School of Public Health, University of Toronto, Toronto, Canada. ${ }^{4}$ HPME, Dalla Lana School of Public Health, University of Toronto, Toronto, Canada. ${ }^{5}$ Department of Sports Science and Clinical Biomechanics, University of Southern Denmark, Odense, Denmark. ${ }^{6}$ Nordic Institute of Chiropractic and Clinical Biomechanics, Odense, Denmark. ${ }^{7}$ Intervention \& Implementation Research for Worker Health, Institute of Environmental Medicine, Karolinska Institutet, Stockholm, Sweden. ${ }^{8}$ ELIB - et liv i bevegelse, Oslo, Norway. ${ }^{9}$ Department for Regional Health Research, University of Southern Denmark, Odense, Denmark. ${ }^{10}$ Department de Chiropractique, Université du Québec à Trois-Rivières, Trois-Rivières, Canada. ${ }^{11}$ Department of Chiropractic, Faculty of Science and Engineering, Macquarie University, Sydney, Australia.

${ }^{12}$ Department of Physical Therapy, Faculty of Rehabilitation Medicine, University of Alberta, Edmonton, Canada. ${ }^{13}$ Canadian Memorial Chiropractic College, Toronto, Canada. ${ }^{14}$ Department of Human Health \& Nutritional Sciences, University of Guelph, Guelph, Canada. ${ }^{15}$ Rebecca MacDonald Centre, Mount Sinai Hospital, Toronto, Canada. ${ }^{16}$ Department of Health

C C The Author(s). 2021 Open Access This article is licensed under a Creative Commons Attribution 4.0 International License, which permits use, sharing, adaptation, distribution and reproduction in any medium or format, as long as you give appropriate credit to the original author(s) and the source, provide a link to the Creative Commons licence, and indicate if changes were made. The images or other third party material in this article are included in the article's Creative Commons licence, unless indicated otherwise in a credit line to the material. If material is not included in the article's Creative Commons licence and your intended use is not permitted by statutory regulation or exceeds the permitted use, you will need to obtain permission directly from the copyright holder. To view a copy of this licence, visit http://creativecommons.org/licenses/by/4.0/ The Creative Commons Public Domain Dedication waiver (http://creativecommons.org/publicdomain/zero/1.0/) applies to the data made available in this article, unless otherwise stated in a credit line to the data. 
Research Methods, Evidence \& Impact, Faculty of Health Sciences, McMaster University, Hamilton, Canada. ${ }^{17}$ School of Physical \& Occupational Therapy, McGill University, Montreal, Canada. ${ }^{18}$ Faculty of Medicine, Memorial University of Newfoundland, St. John's, Canada. ${ }^{19}$ Faculty of Life Sciences and Education University of South Wales, Cardiff, UK. ${ }^{20}$ Institut Franco-Européen de Chiropraxie, Ivry-Sur-Seine, France. ${ }^{21}$ Faculty of Kinesiology, University of New Brunswick, Fredericton, Canada. ${ }^{22}$ Department of Chiropractic Medicine, Faculty of Medicine, University of Zurich \& Balgrist University Hospital, Zurich, Switzerland. ${ }^{23}$ Department of Anesthesiology, University of Kansas Medical Center, Kansas City, USA. ${ }^{24}$ RMIT University, Melbourne, Australia. ${ }^{25}$ College of Science, Health, Engineering and Education, Murdoch University, Murdoch, Australia. ${ }^{26} \mathrm{AECC}$ University College, Bournemouth, UK. ${ }^{27}$ Spine Center of Southern Denmark, University Hospital of Southern Denmark, Middelfart, Denmark. ${ }^{28}$ Faculty of Kinesiology \& Recreation Management University of Manitoba, Winnipeg, Canada. ${ }^{29}$ School of Chiropractic, University of

Bridgeport, Bridgeport, USA. ${ }^{30}$ Faculty of Medicine, School of Population and Public Health, University of British Columbia, Vancouver, Canada. ${ }^{31}$ School of Health, Medical and Applied Sciences, CQ University, Sydney, Australia.

${ }^{32}$ Stanford University School of Medicine, Stanford University, Stanford, USA.

${ }^{33}$ School of Sport and Health Sciences, University of Central Lancashire,

Preston, England.

Published online: 08 March 2021

\section{Reference}

1. Côté $P$, et al. The global summit on the efficacy and effectiveness of spinal manipulative therapy for the prevention and treatment of non-

musculoskeletal disorders: a systematic review of the literature. Chiropr Man Therap. 2021;19:348. https://doi.org/10.1186/s12998-021-00362-9. 